\title{
Étude phytoécologique des adventices dans les agro- écosystèmes élaeicoles de la Mé et de Dabou, en basse Côte d'Ivoire
}

\author{
Karidia Traoré1, Crépin B. Péné ${ }^{2}$ \\ 1. Université J Lorougnon-Guédé de Daloa/UFR Agro-Foresterie et Environnement, 01 BP V44 Daloa 01, Côte \\ d'Ivoire \\ 2. Direction R\&D SUCAF-CI/SOMDIAA, 22 rue des Carrossiers, 01 BP1967 Abidjan 01, Côte d'Ivoire. \\ Email: bpene@sucafci.somdiaa.com
}

Original submitted in on $8^{\text {th }}$ August 2016. Published online at www.m.elewa.org on 31st August 2016 http://dx.doi.org/10.4314/jab.v104i1.13

\section{RÉSUMÉ}

Objectif : Réaliser un inventaire de la flore adventice de quatre agro-écosystèmes de palmier à huile afin de caractériser sa distribution spatiale suivant des descripteurs agro-écologiques pertinents.

Méthodes et résultats : L'étude a donné lieu à un échantillonnage stratifié dans quatre agro-écosystèmes élaeicoles à La Mé et à Dabou, en basse Côte d'Ivoire (respectivement forestière et savanicole). L'échantillonnage a été basé sur 4 descripteurs agro-écologiques comportant au total 17 classes. L'analyse globale des profils écologiques qui en a résulté, a montré que la phytocénose inventoriée comportait 3 groupes d'adventices dont deux étaient caractéristiques des localités étudiées. Le site et le précédent cultural sont apparus les deux principaux descripteurs écologiques efficaces permettant d'expliquer la distribution des adventices. L'analyse des profils de fréquences corrigées a permis, en outre et de façon plus fine, de distinguer 3 groupes écologiques relativement aux différentes classes du descripteur « agro-écosystème » et 4 autres par rapport aux classes du descripteur « précédent cultural».

Conclusion et recommandation: Trois groupes d'espèces adventices ont été déterminés dont deux étaient caractéristiques des localités étudiées. Le 1er comportait deux espèces (Lantana camara et Thaumatococcus daniellii) indicatrices des agro-écosystèmes de La Mé, en zone forestière. Le 2è comprenait 6 espèces (Megastachya mucronata, Polygala multiflora, Anthocleista djalonensis, Asystasia gangetica, Borreria latifolia et Clerodendrum splendens) indicatrices des agro-écosystèmes de Dabou, en zone de savane incluse. Le 3è groupe était constitué d'espèces présentant plutôt une tendance ubiquiste. Le site et le précédent cultural sont apparus les deux principaux descripteurs écologiques efficaces permettant d'expliquer la distribution des adventices.

Mots clés : palmier à huile, adventice, profil écologique, descripteur, zone forestière, savane incluse. 
Phyto-ecological survey of Weeds in Oil Palm Agro-ecosystems of La Mé and Dabou, in Southern Côte d'Ivoire

\begin{abstract}
Objective : The purpose of the study was to characterize the distribution of weed species in palm oil following relevant agro-ecological factors.

Methods and results : A floristic survey of weeds was carried out on four oill palm agro-ecosystems of La Mé and Dabou, in southern Ivory Coast. The sampling procedure used involved 4 ecological factors split into as much as 17 sub-factors. The global analysis of ecological profiles showed that the weed population surveyed was composed of 3 groupings from which two were site-specific species. The agro-ecosystem as well as the preceding crop appeared as main effective ecological factors which could explain weed distribution. Furthermore, the analysis of corrected frequency profiles shows 3 ecological groupings regarding the agroecosystem factor and 4 others with respect to the preceeding crop factor.

Conclusion and recommendation: Three groupings of weed species were determined from which two were site specific of both locations investigated. The first one was composed of two species, namely Lantana camara and Thaumatoccocus daniellii, as indicators of La Mé agro-ecosystems in the forest zone. The second one was composed of 6 species, i.e. Megastachya mucronata, Polygala multiflora, Anthocleista djalonensis, Asystasia gangetica, Borreria latifolia et Clerodendrum splendens, as indicators of Dabou agro-ecosystems in the savannah zone. The third group involved rather ubiquity-oriented species. The location and the preceding crop stood as main effective ecological descriptors which could explain the distribution of weed species.
\end{abstract}

Keywords : oil palm, weed, ecological profile, descriptor, forest zone, included-savannah, Côte d'Ivoire.

\section{INTRODUCTION}

Les systèmes de cultures développés jusque là dans les pays d'Afrique sub-saharienne ont été orientés principalement vers une productivité accrue, souvent au détriment de l'équilibre écologique. La gestion de l'enherbement devient l'un des principaux facteurs permettant l'intensification des cultures et la valorisation de nouvelles techniques (Le Bourgeois, 1993). Pourtant, la lutte contre les mauvaises herbes se résume trop souvent à une action défensive visant à protéger une culture, plutôt qu'à une action offensive visant à maîtriser le développement d'un organisme suffisamment connu pour utiliser les méthodes les plus appropriées à une situation donnée. Aussi, le maintien de l'enherbement en dessous d'un seuil de nuisibilité (Caussanel, 1989) n'est-il pas toujours obtenu. De plus, l'utilisation répétée d'herbicides favorise la sélection d'espèces non sensibles devenant de plus en plus dommageables aux cultures (Déat et Touré, 1983). Cela est en grande partie dû à la méconnaissance de ces enherbements quant à l'écologie et la biologie des espèces qui les composent. Or, la connaissance de la composition de cette flore et de son évolution sous l'effet des facteurs environnementaux et/ou agronomiques est un préalable indispensable à toute amélioration des techniques de lutte contre les mauvaises herbes (Barralis et Chadoeuf, 1980 ; Karar et al, 2005). Cela nécessite que l'étude des adventices soit abordée en amont des recherches finalisées concernant la lutte proprement dite (Maillet, 1992). Les études relatives aux groupements végétaux caractéristiques des milieux se sont développées à partir des années 1970 (Barralis, 1976). A partir des années 1980, un intérêt croissant a été porté à l'écologie des mauvaises herbes et à leurs relations avec la culture (Aman Kadio, 1978 ; Akoundu, 1987 ; Barberi et al, 1997 ; Debaeke, 1987 ; Maillet, 1992). Toutefois, peu de travaux dans ce domaine ont été consacrés à l'Afrique sub-saharienne, où les premières études réalisées remontent à la fin des années 1980 avec les travaux d'Hoffmann (1986) et de Fontanel (1987) sur la comparaison de la flore des terroirs. Les études à vocation régionale ont concerné les cultures céréalières au Burkina Faso (Traoré, 1991), les systèmes de cultures à base de cotonnier au Cameroun (Le Bourgeois, 1993) et en Côte d'Ivoire (Ipou, 2005), la canne à sucre et l'ananas en Côte 
d'Ivoire (Boraud, 2000, Mangara et al, 2010). La présente étude vise à mettre en évidence les relations existant entre les mauvaises herbes et le

\section{MATÉRIELS ET MÉTHODES}

Sites de l'étude : L'étude a été conduite dans 4 agroécosystèmes élaeicoles constitués, d'une part, de parcelles pilotes des stations de recherches sur le palmier à huile (Elaeis guineensis L. Jacq.) du CNRA à La Mé et à Dabou et, d'autre part, de plantations villageoises environnantes.

Domaine de La Mé : Le domaine élaeicole de La Mé, comprenant les parcelles pilotes du CNRA et les plantations villageoises de palmier à huile de La Mé, se situe en retrait du Golfe de Guinée, dans la zone de forêt ombrophile, à une trentaine de $\mathrm{km}$ au Nord-Est de la ville d'Abidjan, sur l'axe routier conduisant à Alépé $\left(5^{\circ} 26^{\prime} \mathrm{N}\right.$, $3^{\circ} 50^{\prime} \mathrm{E} ; 23 \mathrm{~m}$ a.n.m.). II est marqué par un climat équatorial de transition (Péné et Assa, 2003) qui se caractérise par deux saisons de pluies distinctes (avriljuillet et octobre-novembre) intercalées alternativement par deux saisons sèches (décembre-mars et aoûtseptembre). La pluviométrie moyenne annuelle est de l'ordre de $1500 \mathrm{~mm}$. Les $3 / 4$ des sols du domaine sont constitués par des sables tertiaires sur les plateaux et les versants qui sont le siège d'une intense lixiviation d'éléments minéraux et d'un appauvrissement en argile sous l'effet des pluies d'orage (Énomyo, 1985). Le reste (1/4) est constitué de sols hydromorphes (sols organiques, tourbe, gley) dans les bas-fonds.

Domaine de Dabou : Avec une superficie de 5000 ha, ce domaine comprend les plantations pilotes CNRA de la Station R. Michaux et les plantations villageoises environnantes de palmier à huile de Dabou. II est situé à une soixantaine de kilomètres à l'Ouest de la ville d'Abidjan sur l'axe routier reliant les villes de Dabou et de Grand-Lahou ( $5^{\circ} 20^{\prime} \mathrm{N} ; 4^{\circ} 20^{\prime} \mathrm{W}$; 5-20 m a.n.m.). II se trouve dans une végétation originale, qu'est la savane, au milieu dans quatre agro-écosystèmes élaeicoles à La Mé et à Dabou, en basse Côte d'Ivoire.

sein de la zone forestière de la basse Côte d'Ivoire (Caliman, 1990). Ce domaine se caractérise par un climat subéquatorial à deux saisons de pluies : l'une centrée sur le mois de juin, l'autre plus réduite occupant les mois d'octobre et de novembre. La pluviométrie moyenne annuelle est de l'ordre de $1700 \mathrm{~mm}$. Les sols en place sont essentiellement de type sablo-argileux avec une tendance plus argileuse dans les zones de pentes accentuées. Le phénomène dominant dans ces sols est l'entraînement des particules par lessivage qui est accentué par la mise en culture (Roose et al, 1981).

Échantillonnage : L'inventaire floristique a été effectué, de janvier à août 2004, dans des plantations pilotes du CNRA et des plantations villageoises de chaque localité à travers des placettes d'observations de $7 \mathrm{~m}^{2}$, suivant la méthode d'échantillonnage stratifié, avec comme descripteurs (ou variables) 5 paramètres écologiques (classe d'âge de la palmeraie, toposéquence, entité culturale, précédent cultural, agroécosystème). Chaque variable comportait 3 à 4 niveaux, soit 17 strates en tout, le nombre total de relevés pour les deux localités étant de 397 dont 260 dans les parcelles pilotes du CNRA et de 137 dans les plantations villageoises (tableau 1). La taille de chaque placette était réduite à la superficie des ronds autour des palmiers $\left(7 \mathrm{~m}^{2}\right)$, tandis que dans les interlignes et les andains, elle mesurait $1 \mathrm{~m}$ de largeur sur $7 \mathrm{~m}$ de longueur. Les relevés ont porté sur trois placettes d'observations par classe de descripteur. Le nombre moyen de relevés par classe de descripteur est calculé sur les trois placettes. L'identification des espèces relevées, suivant la clé dichotomique de détermination des familles, a été assurée avec l'appui du Centre National de Floristique de l'Université d'Abidjan-Cocody.

Tableau 1: Variables écologiques d'échantillonnage et leurs strates respectives.

\begin{tabular}{l|l|l|l|l|l}
\hline \multirow{2}{*}{} & \multicolumn{5}{|c}{ Descripteurs écologiques } \\
\cline { 2 - 6 } & Classe d'âge & Toposéquence & Entité culturale & Précédent cultural & Agroécosystème \\
\hline Strates & Age1 & $\mathrm{Bf}$ & And & Fo & CNRALM \\
& Age2 & $\mathrm{Ve}$ & Int & Ja & PVLM \\
& Age3 & $\mathrm{Pl}$ & Ron & $\mathrm{Pa}$ & CNRADB \\
& & & $\mathrm{Sa}$ & PVDB \\
\hline
\end{tabular}

Age1 : parcelles de 1 à 5 ans; Age2 : parcelles de 6 à 10 ans ; Age3 : parcelles de 11 à 20 ans

$\mathrm{Bf}$ : bas-fond ; Ve : versant ; PI : plateau, And : endain ; Int : interligne ; Ron : rond ; Fo : forêt ; Sa : savane, Ja : jachère ; Pa : palmier. CNRALM : Station CNRA La Mé ; PVLM : plantations villageoises à La Mé ; CNRADB : Station CNRA Dabou ; PVDB : plantations villageoises à Dabou. 
Fréquences absolue et relative d'une espèce : Les relevés ont consisté, dans chaque placette d'observations, à noter la présence des espèces s'y trouvant, indépendamment du nombre d'individus qu'elles comportaient. La fréquence absolue de chaque espèce est égal au nombre total de ses présences dans l'ensemble des relevés effectués sur les 4 agroécosystèmes élaeicoles. La fréquence relative (Frel) d'une espèce végétale donnée se déduit de sa fréquence absolue (ou nombre de relevés) où elle est présente (Fabs) et du nombre total (NR) de relevés effectués comme suit :

Frel $(\%)=100 \times$ Fabs $/$ NR

Cortège floristique analysé : Sur l'ensemble des 264 espèces d'adventices inventoriées dans les 4 agroécosystèmes à La Mé et à Dabou, seulement 26 ont été prises en compte dans l'analyse factorielle des correspondances (AFC) en raison des contraintes de mémoire quant au traitement des données recueillies à l'aide du logiciel Statistica (version 6). Pour ce faire, seuls les individus dont les fréquences relatives sont supérieures ou égales à $10 \%$ ont été pris en compte dans l'AFC.

Analyse des données phytoécologiques :

Profils écologiques : Un profil écologique est une suite de fréquences (absolues, relatives, pondérées ou corrigées) ordonnées selon les magnitudes du descripteur envisagé. Le profil écologique d'une espèce est un ensemble d'indices de fréquence attachés aux classes d'un descripteur du milieu (Daget et Godron, 1982). L'élaboration du profil écologique d'un descripteur du milieu consiste à regrouper les espèces suivant leur affinité pour les différentes modalités du descripteur.

Le profil des fréquences absolues (Fabs) indique le nombre de présences (ou d'absences) d'une espèce d'adventice dans les classes du descripteur envisagé ; Le profil des fréquences relatives d'une espèce $E$ relativement à un descripteur $L$, n'est autre que l'expression en pourcentage du nombre de relevés des individus représentant l'espèce. II s'agit donc d'une pondération, classe par classe, du profil des fréquences absolues par le profil d'ensemble ;

Le Profil des fréquences corrigées (Fcorr) s'obtient en pondérant les fréquences relatives des présences (ou des absences) d'une espèce $E$, dans la classe $K d u$ descripteur $L$, par sa fréquence relative moyenne des présences (ou des absences) dans l'ensemble des relevés. II se justifie par le fait que le profil des fréquences relatives donne des fréquences faibles ou élevées, respectivement, pour les espèces rares ou abondantes ;
Information mutuelle I $(L ; E)$ entre une espèce $E$ et un descripteur écologique $L$ : C'est la quantité d'information apportée par l'espèce $E$ relativement à un descripteur écologique L. Elle est exprimée comme suit (Guillerm, 1971):

$I(L ; E)=\sum_{1} N K U(K) / N R \times \log _{2}[U(K) / N K \times N R / U(E)]+$ $\sum_{1}{ }^{N K V}(K) / N R . \log _{2}[V(K) / N K \times N R / V(E)]$

Où :

NK : nombre de classes distinguées pour le descripteur $L$;

$U(E)$ : nombre total de relevés où l'espèce $E$ est présente;

$V(E)$ : nombre total de relevés où l'espèce E est absente ; $U(K)$ : nombre de relevés où l'espèce $E$ est présente dans la classe $\mathrm{K}$ du descripteur ;

$V(E)$ : nombre de relevés où l'espèce $E$ est absente dans la classe $\mathrm{K}$ du descripteur ;

$\mathrm{NR}$ : nombre total de relevés relativement à l'ensemble des espèces et des descripteurs.

L'information mutuelle I ( $L$; $E$ ) est déterminante pour la constitution des groupes d'espèces indicatrices. Sa valeur est d'autant plus élevée que la liaison entre une espèce et un descripteur est fort. Dans chaque groupe, les espèces indicatrices sont classées par ordre décroissant de leurs informations mutuelles. On peut ainsi facilement détecter, à l'intérieur d'un groupe indicateur, des sous-groupes dont les limites sont nettement marquées. En effet, la limite supérieure d'un groupe ou sous-groupe est toujours indiquée par une espèce dont l'information est supérieure à celle de l'espèce qui la succède et qui constitue la limite inférieure du groupe ou sous-groupe précédent.

Analyse factorielle des correspondances: Elle s'est avérée très adaptée aux études phytosociologiques et phytoécologiques en ce sens qu'elle permettait d'obtenir une vision synthétique des liaisons entre espèces et facteurs du milieu (Lebreton et al, 1991 ; Sabatier et al, 1989). Elle donne la possibilité de résumer, en quelques dimensions importantes (ou axes factoriels indépendants), la plus grande variabilité de la matrice des données. On peut alors représenter variables et individus dans un même espace de dispersion et connaître la quantité d'information expliquée par ces quelques axes factoriels indépendants (Legendre et Legendre, 1984 cités par Le Bourgeois, 1993), rendant ainsi compte du maximum de covariance entre les descripteurs. II s'agit donc d'une approche globale qui dégage les relations essentielles existant entre la végétation et le milieu.

L'analyse fait intervenir deux matrices de données $Y$ (n, $p)$ et $X(n, q)$ où $n=397$ est le nombre de relevés, $p=26$, le nombre d'espèces et $q=17$, celui des descripteurs du 
milieu. La matrice $Y$ contient les variables d'intérêt direct (les espèces) en indice de présence et la matrice $X$, les variables caractérisant la structure (descripteurs du milieu) supposées influencer les variables de Y, c'est-àdire la répartition des espèces. L'analyse nécessite deux étapes comme rapporté par Le Bourgeois (1993) :

- La première consiste à calculer $p$ régressions multiples simultanées dans une métrique $D$ de la matrice $Y$ des espèces sur la matrice $X$ des descripteurs. Ainsi, toute espèce $y_{i}$ peut s'exprimer comme une combinaison linéaire des descripteurs :

$\mathrm{y}_{\mathrm{i}}=$ cste $+\alpha \mathrm{x}_{1}+\beta \mathrm{x}_{2}+\ldots+\delta \mathrm{x}_{\mathrm{q}}+\varepsilon_{\mathrm{i}}$

C'est-à-dire :

$y_{i}=\hat{y}_{i}+\varepsilon_{i}$

Où ŷy est une estimation de $y_{i}$ et $\varepsilon_{i}$ le résidu.

On peut ainsi constituer une nouvelle matrice PY (des estimations $\hat{y}_{i}$ des $\left.y_{i}\right)$ correspondant à la part expliquée de $Y \operatorname{par} X\left[P=X(X D X)^{-1} X D\right]$;
- La seconde étape revient à effectuer une analyse factorielle sur la matrice PY des estimations des $y_{\text {i. }}$. Les axes factoriels étant des combinaisons linéaires des estimations des espèces et des descripteurs, les covariances sont maximisées. En utilisant une mesure moyenne des relations entre les espèces et les descripteurs, l'analyse factorielle amplifie la dissymétrie naturelle des relations inhérentes aux études d'interactions «Structure de la végétation/ Environnement». Dans le même temps, cette caractéristique augmente la robustesse de l'analyse et sa validité (Lebreton et al, 1991). Pour faciliter l'analyse, le nom de chaque espèce a été codé en référence au code Bayer (1992) utilisé par Le Bourgeois (1993). En effet, une espèce est représentée par les trois premières lettres du genre et les deux premières lettres de l'épithète spécifique (tableau 2).

Tableau 2 : Liste des espèces prises en compte dans l'AFC sur l'ensemble des relevés dans les différents sites des deux localités.

\begin{tabular}{|c|c|c|c|}
\hline Code & Espèce d'adventice & Famille botanique & Type biologique \\
\hline Ageco & Ageratum conyzoïdes $\mathrm{L}$. & Asteraceae & Thérophyte \\
\hline Antdj & Anthocleista djalonensis A.Chev. & Loganiaceae & Microphanérophyte \\
\hline Asyga & Asystasia gangetica (L.) T.Anders. & Acanthaceae & Nanophanérophyte \\
\hline Borla & Borreria latifolia (Aublet.) K.Schum. & Rubiaceae & Thérophyte \\
\hline Cenpu & Centrosema pubescens Benth. & Fabaceae & Liane microphanérophyte \\
\hline Chrod & Chromolaena odorata (L.) R.King \& H.Rob. & Asteraceae & Nanophanérophyte \\
\hline Clesp & Clerodendrum splendens G.Don & Verbenaceae & Liane microphanérophyte \\
\hline Crohi & Croton hirtus L'herit & Euphorbiaceae & Nanophanérophyte \\
\hline Desad & Desmodium adscendens (Sw.) DC. & Fabaceae & Chaméphyte \\
\hline Emipr & Emilia praetermissa & Asteraceae & Thérophyte \\
\hline Hetro & Heterotis rotundifolia (Sm.) Jacq. & Melastomataceae & Chaméphyte \\
\hline Ipoin & Ipomoea involucrata P.Beauv. & Convolvulaceae & Thérophyte \\
\hline Lanca & Lantana camara L. & Verbenaceae & Liane microphanérophyte \\
\hline Maral & Mariscus alternifolius Vahl & Cyperaceae & Hémicryptophyte \\
\hline Marfl & Mariscus flabelliformis Kunth & Cyperaceae & Hémicryptophyte \\
\hline Megm & Megastachya mucronata (Poir.) P.Beauv. & Poaceae & Thérophyte \\
\hline Nepbi & Nephrolepis biserrata (Sw.) Schott. & Davalliaceae & Hémichriptophyte Épiphyte \\
\hline Oplbu & Oplismenus burmannii (Retz.) P.Beauv. & Poaceae & Chaméphyte \\
\hline Panla & Panicum laxum Sw. & Poaceae & Thérophyte \\
\hline Phafa & Phaulopsis falcisepala C.B.Cl. & Acanthaceae & Nanophanérophyte \\
\hline Phyam & Phyllanthus amarus Schum \& Thonn. & Euphorbiaceae & Nanophanérophyte \\
\hline Physc & Phymatodes scolopendria (Burn.) Ching & polypodiaceae & Épiphyte \\
\hline Polmu & Polygala multiflora Poir. & Polygalaceae & Thérophyte \\
\hline Sabve & Sabicea venosa Benth. & Rubiaceae & Liane nanophanérophyte \\
\hline Secaf & Secamone afzelii (Schult.) K.Schum. & Asclepiadaceae & Liane microphanérophyte \\
\hline Thada & Thaumatococcus daniellii (Benn.) Benth. & Marantaceae & Géophyte rhyzomateux \\
\hline Total & 26 & 16 & 9 \\
\hline
\end{tabular}


RÉSULTATS ET DISCUSSION

Profils de fréquences et corrélation entre les espèces et les descripteurs écologiques : La corrélation entre les espèces d'adventice et les descripteurs écologiques, effectuée à partir des profils de fréquence relative (tableau 3), est généralement quasiment nulle et non significative, hormis deux descripteurs qui se révèlent efficaces (site et précédent cultural). En effet, les modalités de ceux-ci contribuent à expliquer de façon notable (au seuil de $1 \%$ ) la répartition des adventices dans les différents agro-écosystèmes étudiés. L'espèce Chromolaena odorata est de loin la plus fréquente $(76,6$ $\%)$, suivie de Nephrolepis biserrata (44,3\%), Panicum laxum $(43,8)$ et Oplismenus burmannii $(42,3 \%)$. Deux espèces se révèlent particulièrement moins fréquentes : Lantana camara (10\%) et Polygala multiflora (10,6\%).

Tableau 3 : Profils de fréquences des espèces d'adventice suivant les descripteurs efficaces et profils d'ensemble.

\begin{tabular}{|c|c|c|c|c|c|c|c|c|c|c|}
\hline \multirow{3}{*}{ Espèces d'adventice } & \multicolumn{8}{|c|}{ Descripteurs écologiques efficaces (fréquences absolues) } & \multirow{2}{*}{\multicolumn{2}{|c|}{$\begin{array}{c}\text { Profils } \\
\text { d'ensemble }\end{array}$}} \\
\hline & \multicolumn{4}{|c|}{ Précédents culturaux } & \multicolumn{4}{|c|}{ Agro-écosystèmes } & & \\
\hline & $\mathrm{Pa}$ & $\mathrm{Ja}$ & $\mathrm{Sa}$ & Fo & $\begin{array}{l}\text { CNRA } \\
\text { La Mé }\end{array}$ & $\begin{array}{c}\text { PV } \\
\text { La Mé }\end{array}$ & CNRA Dabou & PV Dabou & $\begin{array}{c}\mathrm{Fr} \\
\mathrm{abs}\end{array}$ & Fr rel $(\%)$ \\
\hline Chromolaena odorata & 87 & 89 & 50 & 31 & 66 & 57 & 115 & 66 & 304 & 76,57 \\
\hline Nephrolepis biserrata & 50 & 40 & 39 & 47 & 49 & 36 & 53 & 37 & 176 & 44,33 \\
\hline Panicum laxum & 52 & 41 & 26 & 55 & 77 & 45 & 27 & 25 & 174 & 43,83 \\
\hline Oplismenus burmannii & 61 & 50 & 23 & 34 & 51 & 25 & 70 & 22 & 168 & 42,32 \\
\hline Heterotis rotundifolia & 40 & 35 & 13 & 16 & 24 & 38 & 59 & 13 & 134 & 33,75 \\
\hline Centrosema pubescens & 32 & 47 & 15 & 7 & 18 & 5 & 61 & 17 & 101 & 25,44 \\
\hline Phaulopsis falcisepala & 27 & 48 & 18 & 5 & 68 & 7 & 20 & 3 & 98 & 24,69 \\
\hline Emilia praetermissa & 10 & 22 & 37 & 28 & 14 & 25 & 21 & 37 & 97 & 24,43 \\
\hline Desmodium adscendens & 19 & 16 & 14 & 47 & 23 & 40 & 19 & 14 & 96 & 24,18 \\
\hline Mariscus alternifolius & 28 & 27 & 15 & 20 & 35 & 19 & 21 & 15 & 90 & 22,67 \\
\hline Asystasia gangetica & 47 & 35 & 4 & 1 & 4 & 0 & 80 & 3 & 87 & 21,91 \\
\hline Clerodendrum splenden & 22 & 25 & 16 & 7 & 38 & 0 & 17 & 15 & 70 & 17,63 \\
\hline Secamone afzelii & 18 & 25 & 16 & 2 & 24 & 16 & 20 & 1 & 61 & 15,37 \\
\hline Phymatodes scolopendria & 4 & 25 & 18 & 13 & 3 & 10 & 29 & 18 & 60 & 15,11 \\
\hline Croton hirtus & 24 & 4 & 25 & 5 & 2 & 5 & 26 & 25 & 58 & 14,61 \\
\hline Phyllanthus amarus & 11 & 17 & 8 & 22 & 17 & 19 & 14 & 8 & 58 & 14,61 \\
\hline Thaumatococcus daniellii & 29 & 10 & 0 & 16 & 40 & 15 & 0 & 0 & 55 & 13,85 \\
\hline Ipomoea involucrata & 16 & 11 & 16 & 10 & 18 & 1 & 19 & 15 & 53 & 13,35 \\
\hline Mariscus flabelliformis & 12 & 21 & 0 & 22 & 24 & 15 & 14 & 0 & 53 & 13,35 \\
\hline Megastachya mucronata & 16 & 18 & 18 & 0 & 0 & 0 & 34 & 18 & 52 & 13,10 \\
\hline Sabicea venosa & 4 & 13 & 20 & 7 & 1 & 26 & 16 & 1 & 44 & 11,08 \\
\hline Ageratum conyzoïdes & 22 & 4 & 8 & 9 & 15 & 8 & 12 & 8 & 43 & 10,83 \\
\hline Anthocleista djalonensis & 6 & 15 & 16 & 6 & 0 & 6 & 21 & 16 & 43 & 10,83 \\
\hline Borreria latifolia & 17 & 11 & 15 & 5 & 0 & 0 & 21 & 15 & 43 & 10,83 \\
\hline Polygala multiflora & 0 & 0 & 42 & 0 & 0 & 0 & 0 & 42 & 42 & 10,57 \\
\hline Lantana camara & 24 & 6 & 1 & 5 & 18 & 5 & 16 & 1 & 40 & 10,00 \\
\hline
\end{tabular}

PV : plantations villageoises

CNRA : parcelles expérimentales ou pilotes

Fo : forêt ; Sa : savane, Ja : jachère ; Pa : palmier.

Analyse factorielle des correspondances (AFC) : II ressort de l'AFC prenant en compte soit l'ensemble des descripteurs écologiques, soit les deux descripteurs efficaces, que le plan factoriel 1-2 contient l'essentiel de l'information relativement à la répartition des adventices dans les agro-écosystèmes étudiés.

La prise en compte, d'une part, des modalités de l'ensemble des descripteurs et, d'autre part, de celles des 
deux descripteurs efficaces (site et précédent cultural) dans l'AFC permet de déterminer 3 groupes d'espèces d'adventices (figures 1 et 2). Le groupe 1, constitué de 2 espèces (Lantana camara et Thaumatococcus daniellii), semble être indicateur des agro-écosystèmes de La Mé, en zone forestière. À l'opposée, le groupe 2 qui referme 6 espèces (Megastachya mucronata, Polygala multiflora, Anthocleista djalonensis, Asystasia gangetica, Borreria latifolia, Clerodendrum splendens) semble être caractéristique des agro-écosystèmes de Dabou, en zone de savane incluse. Le groupe 3, constitué par les 18 espèces restantes (Centrosema pubescens, Chromolaena odorata, Croton hirtus, Desmodium adscendens, Emilia praetermissa, Heterotis rotundifolia, Ipomoea involucrata, Mariscus alternifolius, Mariscus flabelliformis), semble présenter une tendance ubiquiste.

2D Plot of Column Coordinates; Dimension: $1 \times 2$ Input Table (Rows x Columns): 43 x 43 (Burt Table)

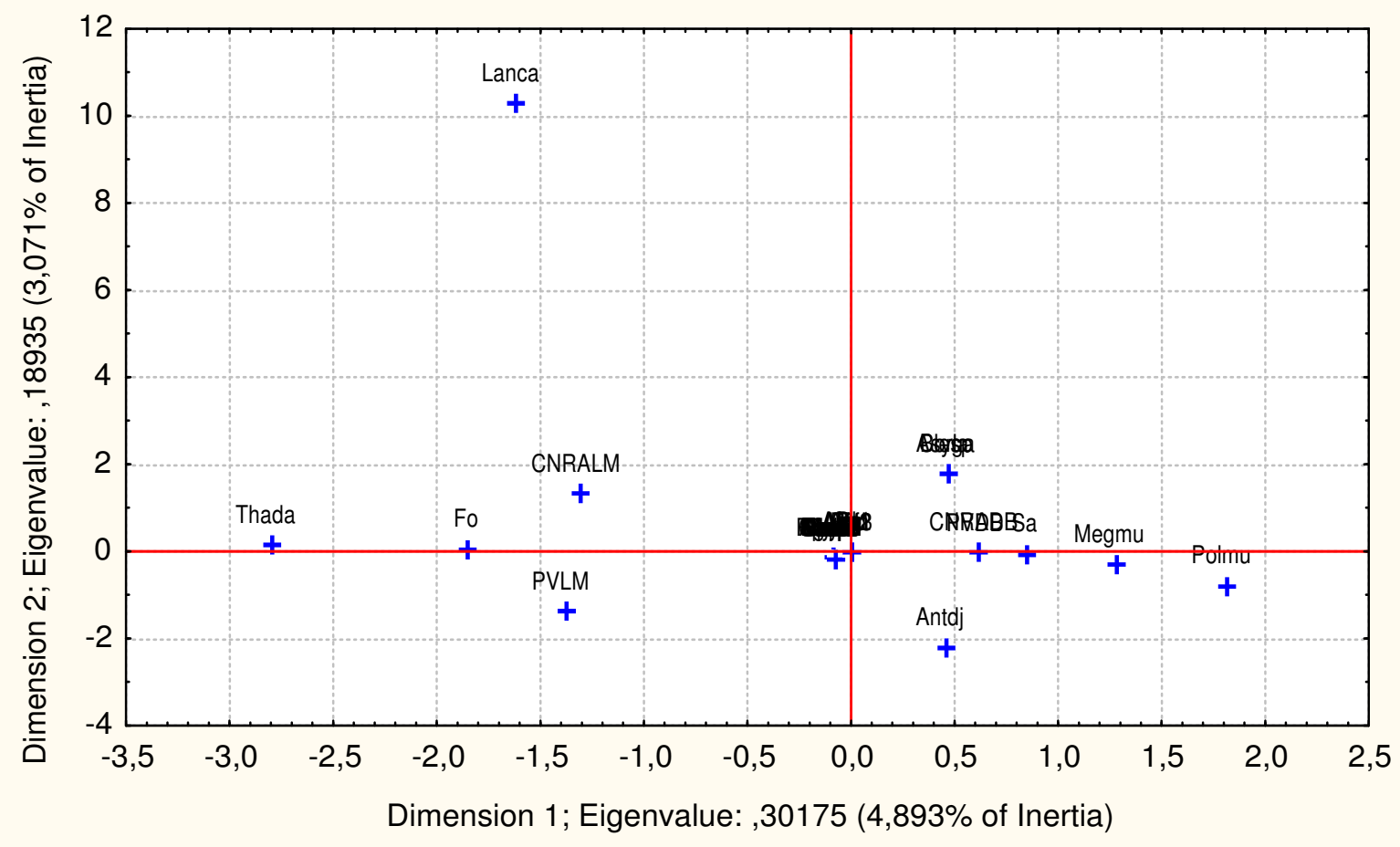

Figure 1 : Projection des individus (espèces) et des modalités de l'ensemble des six descripteurs écologiques (âge palmeraie, toposéquence, entité culturale, précédent cultural, site) sur les axes 1 et 2 de l'AFC. 
2D Plot of Column Coordinates; Dimension: $1 \times 2$

Input Table (Rows x Columns): 34 × 34 (Burt Table)

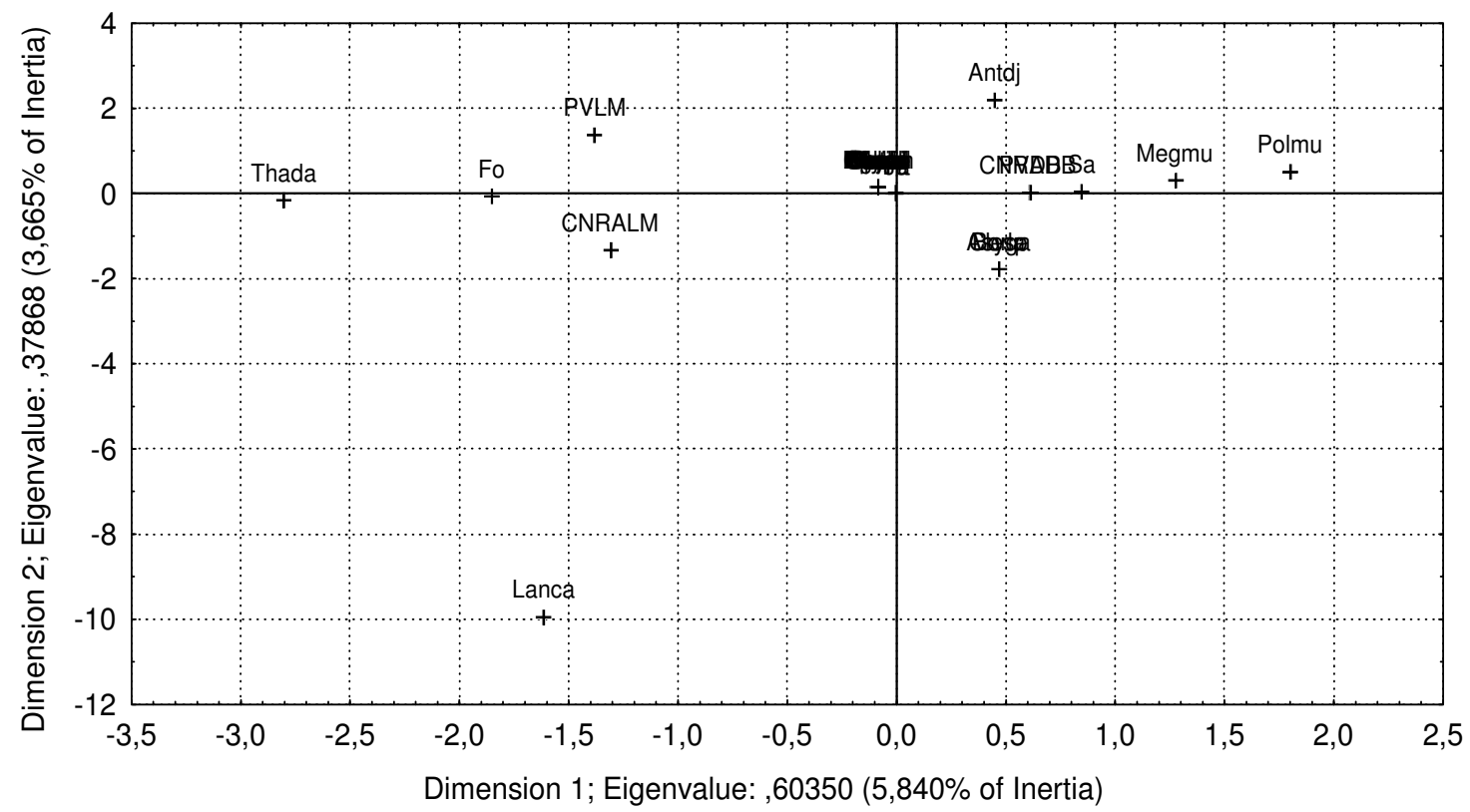

Figure 2 : Projection des individus (espèces) et des modalités de deux descripteurs écologiques efficaces (site et précédent cultural) sur les axes 1 et 2 de l'AFC.

Analyse des profils de fréquences corrigées suivant les descripteurs efficaces: La détermination des groupes écologiques à partir des valeurs d'information mutuelle exclut, par définition, les espèces pour lesquelles au moins une fréquence corrigée est nul (tableau 4). L'AFC a montré que ces espèces étaient indicatrices relativement aux descripteurs écologiques efficaces. Trois groupes écologiques s'observent par rapport aux différentes classes du descripteur « agroécosystème " (tableau V): Les premier et second groupes comportent chacun une espèce d'adventice, soit Phaulopsis falcisepala et Sabicea venosa, respectivement. Phaulopsis falcisepala est une espèce tendant à pousser préférentiellement dans le périmètre du CNRA à La Mé, tandis que Sabicea venosa semble se développer préférentiellement dans les plantations villageoises de La Mé. Le 3è groupe comporte les 17 espèces ci-après : Centrosema pubescens, Desmodium adscendens, Croton hirtus, Secamone afzelii, Clerodendrum splenden, Panicum laxum, Phymatodes scolopendria, Chromolaena odorata, Lantana camara, Phyllanthus amarus, Emilia praetermissa, Ipomoea involucrata, Ageratum conyzoïdes, Mariscus alternifolius, Oplismenus burmannii, Heterotis rotundifolia et Nephrolepis biserrata. Parmi ces espèces, Desmodium adscendens et Phyllanthus amarus tendent à prédominer dans les plantations villageoises de La Mé avec des fréquences corrigées de présences respectives de 263 et 232. 
Tableau 4 : Profils de fréquences corrigées des présences d'espèces relativement aux descripteurs efficaces.

\begin{tabular}{|c|c|c|c|c|c|c|c|c|}
\hline \multirow{3}{*}{ Espèces d'adventice } & \multicolumn{8}{|c|}{ Descripteurs efficaces } \\
\hline & \multicolumn{4}{|c|}{ Agro-écosystèmes } & \multicolumn{4}{|c|}{ Précédents culturaux } \\
\hline & $\begin{array}{l}\text { CNRA } \\
\text { La Mé }\end{array}$ & $\begin{array}{c}\text { PV } \\
\text { La Mé }\end{array}$ & CNRA Dabou & $\begin{array}{c}\text { PV } \\
\text { Dabou }\end{array}$ & Palmeraie & Jachère & Savane & Forêt \\
\hline Ageratum conyzoïdes & 103 & 114 & 88 & 59 & 149 & 34 & 100 & 104 \\
\hline Anthocleista djalonensis & 0 & 85 & 154 & 117 & 41 & 129 & 200 & 69 \\
\hline Asystasia gangetica & 14 & 0 & 290 & 11 & 158 & 149 & 25 & 6 \\
\hline Borreria latifolia & 48 & 0 & 154 & 110 & 115 & 95 & 187 & 0 \\
\hline Centrosema pubescens & 53 & 30 & 190 & 53 & 92 & 173 & 90 & 24 \\
\hline Chromolaena odorata & 64 & 114 & 119 & 68 & 834 & 109 & 118 & 99 \\
\hline Clerodendrum splenden & 170 & 8 & 65 & 65 & 88 & 127 & 117 & 47 \\
\hline Croton hirtus & 9 & 55 & 124 & 153 & 106 & 22 & 203 & 37 \\
\hline Desmodium adscendens & 73 & 263 & 64 & 37 & 60 & 64 & 81 & 251 \\
\hline Emilia praetermissa & 28 & 111 & 119 & 108 & 21 & 59 & 145 & 101 \\
\hline Heterotis rotundifolia & 53 & 173 & 139 & 30 & 87 & 97 & 52 & 170 \\
\hline Ipomoea involucrata & 83 & 9 & 83 & 139 & 69 & 60 & 126 & 73 \\
\hline Lantana camara & 133 & 76 & 126 & 8 & 175 & 56 & 13 & 62 \\
\hline Mariscus alternifolius & 119 & 133 & 76 & 43 & 94 & 115 & 92 & 114 \\
\hline Mariscus flabelliformis & 134 & 173 & 83 & 0 & 66 & 147 & 0 & 187 \\
\hline Megastachya mucronata & 0 & 11 & 298 & 11 & 85 & 121 & 175 & 0 \\
\hline Nephrolepis biserrata. & 83 & 126 & 95 & 67 & 82 & 85 & 119 & 133 \\
\hline Oplismenus burmannii & 81 & 77 & 135 & 54 & 98 & 102 & 68 & 93 \\
\hline Panicum laxum & 110 & 120 & 74 & 62 & 66 & 66 & 61 & 119 \\
\hline Phaulopsis falcisepala & 205 & 44 & 64 & 10 & 80 & 182 & 16 & 101 \\
\hline Phyllanthus amarus & 71 & 232 & 76 & 43 & 55 & 109 & 74 & 188 \\
\hline Phymatodes scolopendria & 15 & 93 & 155 & 96 & 20 & 157 & 164 & 109 \\
\hline Polygala multiflora & 0 & 0 & 0 & 315 & 0 & 0 & 536 & 0 \\
\hline Sabicea venosa & 77 & 294 & 76 & 6 & 22 & 89 & 10 & 239 \\
\hline Secamone afzelii & 116 & 160 & 103 & 5 & 86 & 152 & 9 & 138 \\
\hline Thaumatococcus daniellii & 208 & 182 & 0 & 0 & 148 & 65 & 0 & 139 \\
\hline
\end{tabular}

PV : plantations villageoises

CNRA : parcelles expérimentales ou pilotes

Quatre groupes écologiques s'observent par rapport aux différentes classes du descripteur « précédent cultural » (tableau 6) :

$\checkmark \quad$ le premier renferme 2 espèces (Asystasia gangetica et Desmodium adscendens), Asystasia gangetica se développant préférentiellement lorsque le précédent cultural est une palmeraie (Frcor=158) ou une jachère (Frcor $=149)$. En revanche, Desmodium adscendens pousse préférentiellement lorsque le précédent cultural est une forêt (Frcor=251) ;

$\checkmark$ le second groupe comporte les 16 espèces suivantes: Centrosema pubescens, Phymatodes scolopendria, Chromolaena odorata, Croton hirtus, Anthocleista djalonensis, Phyllanthus amarus, Nephrolepis biserrata, Lantana camara, Ageratum conyzoïdes, Mariscus alternifolius, Clerodendrum splenden, Heterotis rotundifolia, Emilia praetermissa, Phaulopsis falcisepala, Ipomoea involucrata et Oplismenus burmannii. Parmi elles, Centrosema pubescens et Phaulopsis falcisepala tendent à être endémiques lorsque le précédent est la jachère. Croton hirtus (Frcor $=203$ ) et Anthocleista djalonensis (Frcor $=200$ ), quant à elles, tendent à pousser préférentiellement lorsque le précédent est la savane. Quand le précédent est la forêt, les espèces tendant à se développer préférentiellement sont Phyllanthus amarus (Frcor= 188) et Heterotis rotundifolia (Frcorr=170) ;

$\checkmark \quad$ le 3 è groupe renferme 2 espèces (Secamone afzelii et Panicum laxum). Secamone afzelii tend à mieux se développer lorsque le précédent est la jachère (Frcor=152) ou la forêt (Frcor=138). Panicum laxum est, 
par contre, mieux représentée quant le précédent est la forêt (Frcor=119) ;

$\checkmark$ le 4è groupe, comprend une espèce (Sabicea venosa) qui se développe préférentiellement là où le précédent est la forêt (Frcpr= 239).

Influence de l'échantillonnage et de la méthode de traitement des données: L'élaboration des groupes écologiques dépend, d'une façon déterminante, de l'échelle d'observation et de l'échantillonnage. C'est pourquoi, dans cette étude, la taille des placettes d'observations choisie est la même que celle des ronds de sarclage autour de chaque palmier $\left(7 \mathrm{~m}^{2}\right)$. Cette échelle d'observations, conforme à la pratique agricole en matière de désherbage, devrait contribuer fortement à la représentativité des groupes écologiques élaborés. Un groupe écologique est, par définition, un ensemble d'espèces indicatrices présentant la même réaction relativement à un descripteur efficace (ou important). Pour aller au delà de l'expression des grandes tendances écologiques et obtenir des associations plus significatives, il faudrait pouvoir compléter l'échantillonnage et accroître le nombre de relevés (Maillet, 1992). L'attribution d'une espèce à un groupe donné pourrait être alors remise en question. Aussi l'étude des unités de végétation n'est-elle pas une science exacte. Ce qui n'enlève pas pour autant toute valeur prédictive aux relations espèces-milieu. S'il est impossible de prévoir avec exactitude les espèces des différents groupes écologiques, en revanche, il est possible d'établir dans quels groupes écologiques les chances de trouver les éléments des communautés végétales futures sont accrues (Maillet, 1992). Ainsi, la suppression du labour dans l'itinéraire technique d'une culture au profit de l'herbicidage produit des modifications stochastiques dans la composition floristique de la végétation (ne sachant quelles espèces agrestales se maintiendront ni quels apophytes s'installeront). Toutefois, ces modifications ne sont pas aléatoires car il est possible de prévoir de quels groupes écologiques proviendront les espèces et quelles sont les caractéristiques du milieu favorables à leur meilleure végétation. Par ailleurs, l'individualisation des unités de végétation est tributaire de la méthode de traitements des données (Wiegleb, 1989). Aussi est-il important, en l'absence d'élément de choix déterminant a priori, d'utiliser diverses approches dans le traitement des données phytoécologiques (Austin, 1985 ; Jackson et al, 1989). Ainsi par exemple, le calcul de l'information entre chaque espèce d'adventice et les descripteurs efficaces (agro-écosystème et précédent cultural) a permis d'affiner les groupes écologiques déterminés suite à l'analyse globale des données phytoécologiques (figures 1 et 2 ; tableaux 5 et 6 ).

Pratique culturale et répartition des espèces : Les travaux de Maillet (1988) en viticulture ont montré que les perturbations accompagnant l'abandon des labours au profit du désherbage chimique avaient des répercutions rapides sur la composition qualitative et quantitative des communautés de mauvaises herbes. La nature de la relation entre la fréquence et l'abondance des espèces végétales et sa réalité en milieu cultivé, particulièrement importantes en agronomie dans la prévision des risques d'infestation (Forcella et Harvey, 1983 ; Brown, 1984), ont été étudiées sur l'hévéa (Aman Kadio, 1978), la vigne (Maillet, 1992), le coton (Le Bourgeois, 1993; Ipou, 2005), les céréales (Traoré et maillet, 1998), la canne à sucre (Boraud, 2000) et l'ananas (Mangara et al, 2010). En culture annuelle, la phytocénose s'appauvrit généralement rapidement sous la pression des herbicides (Mahn, 1984). L'impact du désherbage chimique se traduit également à l'échelle régionale par une uniformisation des communautés végétales au profit des espèces tolérantes (Tomkins et Grant, 1977 ; Mahn et Helmecke, 1979) et une baisse de la richesse floristique (Hodgson, 1989). Plus rarement, après une phase de réduction initiale de la diversité, celle-ci remonte grâce à la colonisation d'espèces nouvelles non affectées par les herbicides (Malone, 1972). Cela semble être le cas sous certaines cultures pérennes comme le palmier à huile où l'éventail des types biologiques susceptibles de s'implanter est beaucoup plus vaste qu'en cultures annuelles (Maillet, 1988). En monoculture stabilisée, quelle soit pérenne ou annuelle, l'itinéraire technique pratiqué tend à sélectionner un même cortège floristique d'adventices (Traoré et Maillet, 1998). Celui-ci s'adapte ainsi à l'environnement perturbé par les pratiques culturales. Dans le cas du palmier à huile, le caractère pérenne de cette culture, l'ombrage qu'il impose sur le sol au stade adulte et l'absence de labour créent une flore adventice distribuée indépendamment des descripteurs écologiques étudiés. Ces facteurs, combinés à la disponibilité de l'eau du sol pratiquement toute l'année sous palmeraies en climat subéquatorial, réduisent la différenciation des adventices en fonction de l'époque d'observation. Celle-ci semble pourtant influencer notablement la composition floristique des parcelles d'après divers travaux réalisés sur des cultures annuelles (Déat et al, 1980 ; Fontanel, 1987 ; Koffi, 1981 ; Maillet, 1992; Mark, 1983; Traoré, 1991; Traoré et Maillet, 1998). Dans les palmeraies de La Mé, en zone forestière, trois groupes écologiques, définis par rapport au descripteur " agro-écosystème ", se distinguent sur la 
base des différences de pratiques culturales (tableau 5). Les palmeraies de la Station CNRA de La Mé se distinguent de celles du secteur villageois par le fait qu'elles sont mieux gérées sur le plan agronomique (respect du calendrier des désherbages, plante de couverture au jeune âge, fertilisation potassique à l'âge adulte, élagage des couronnes foliaires, andainage des palmes élaguées). L'influence des pratiques culturales sur la distribution de la flore adventice recensée apparaît également à travers les groupes écologiques déterminés par rapport au descripteur « précédent cultural » (tableau 6).

Tableau 5 : Groupes écologiques suivant l'information mutuelle entre les espèces d'adventices et le descripteur « agroécosystème (site) $)$.

\begin{tabular}{|c|c|c|c|c|c|c|}
\hline \multirow{2}{*}{$\begin{array}{l}\text { Espèces } \\
\text { d'adventice }\end{array}$} & \multirow{2}{*}{$\begin{array}{l}\text { Information } \\
\text { mutuelle }\end{array}$} & \multicolumn{4}{|c|}{ Agro-écosystèmes } & \multirow{2}{*}{$\begin{array}{l}\text { Groupes } \\
\text { écologiques }\end{array}$} \\
\hline & & $\begin{array}{l}\text { CNRA } \\
\text { La Mé }\end{array}$ & $\begin{array}{c}\text { PV } \\
\text { La Mé }\end{array}$ & $\begin{array}{l}\text { CNRA } \\
\text { Dabou }\end{array}$ & $\begin{array}{c}\text { PV } \\
\text { Dabou }\end{array}$ & \\
\hline Phaulopsis falcisepala & 6,78 & 205 & 44 & 64 & 10 & I \\
\hline Sabicea venosa & 6,74 & 77 & 294 & 76 & 6 & II \\
\hline Centrosema pubescens & 6,71 & 53 & 30 & 190 & 53 & \\
\hline Desmodium adscendens & 6,71 & 73 & 263 & 64 & 7 & \\
\hline Croton hirtus & 6,69 & 9 & 55 & 124 & 153 & \\
\hline Secamone afzelii & 6,68 & 116 & 160 & 103 & 5 & \\
\hline Clerodendrum splenden & 6,68 & 170 & 8 & 65 & 65 & \\
\hline Panicum laxum & 6,67 & 110 & 120 & 74 & 62 & \\
\hline Phymatodes scolopendria & 6,67 & 15 & 93 & 155 & 96 & \\
\hline Chromolaena odorata & 6,66 & 64 & 114 & 119 & 68 & \\
\hline Lantana camara & 6,65 & 133 & 76 & 126 & 8 & III \\
\hline Phyllanthus amarus & 6,65 & 71 & 232 & 76 & 43 & \\
\hline Emilia praetermissa & 6,64 & 28 & 111 & 119 & 108 & \\
\hline Ipomoea involucrata & 6,63 & 83 & 9 & 83 & 139 & \\
\hline Ageratum conyzoïdes & 6,63 & 103 & 113 & 88 & 59 & \\
\hline Mariscus alternifolius & 6,62 & 119 & 133 & 76 & 43 & \\
\hline Oplismenus burmannii & 6,62 & 81 & 77 & 135 & 54 & \\
\hline Heterotis rotundifolia & 6,61 & 53 & 173 & 139 & 30 & \\
\hline Nephrolepis biserrata & 6,59 & 83 & 126 & 95 & 67 & \\
\hline
\end{tabular}

PV : plantations villageoises

CNRA : parcelles expérimentales ou pilotes

Les espèces d'un groupe écologique donné ont des valeurs d'information mutuelle ne différant pas les unes des autres au seuil de $5 \%$ d'après le test de Student-Fisher.

Descripteurs efficaces et espèces indicatrices: L'importance d'un descripteur dans une analyse écologique n'est autre que son efficacité pour reconnaître des unités phytoécologiques dans l'ensemble des éléments traités. D'une façon générale, les descripteurs les mieux échantillonnés sont en général les plus efficaces. Cela tient au fait que l'échantillonnage est le plus souvent établi en fonction des paramètres présumés déterminants pour la végétation. Une limite à l'utilisation du concept d'efficacité provient du fait qu'on ne disposerait pas encore d'algorithme adapté à la reconnaissance des descripteurs efficaces, leur sélection restant subjective (Daget et Godron, 1982). L'efficacité du descripteur « site » observée dans la présente étude est corroborée par plusieurs travaux antérieurs (Boraud, 2000 ; Le Bourgeois, 1993 ; Traoré, 1991 ; Traoré et Maillet, 1998, Mangara et al, 2010). Ce descripteur est soumis à l'influence combinée de facteurs climatiques (précipitation, température, insolation, hygrométrie) et édaphiques (texture, profondeur, fertilité, topographie). Le climat est le facteur abiotique prépondérant dans la répartition des espèces, suivi du type de sol. En relation avec la pluviométrie, la nature du substrat, la texture et la topographie déterminent les conditions hydriques $\mathrm{du}$ milieu. Quant au descripteur " précédent cultural », il traduit un gradient d'évolution des adventices dans le temps (Le Bourgeois, 1993). 
Tableau 6 : Groupes écologiques suivant l'information mutuelle entre les espèces d'adventices et le descripteur « précédent cultural».

\begin{tabular}{lrrrrrr}
\hline Espèces & Information & \multicolumn{4}{c}{ Précédents culturaux } & \multicolumn{2}{c}{ Groupes } \\
\cline { 3 - 6 } d'adventice & mutuelle & Palmeraie & Jachère & Savane & Forêt & écologiques \\
\hline Asystasia gangetica & 6,76 & 158 & 149 & 25 & 6 & \\
Desmodium adscendens & 6,76 & 60 & 64 & 81 & 251 & I \\
\hline Centrosema pubescens & 6,71 & 92 & 173 & 90 & 24 & \\
Phymatodes scolopendria & 6,71 & 20 & 157 & 164 & 109 & \\
Chromolaena odorata & 6,69 & 83 & 109 & 118 & 99 & \\
Croton hirtus & 6,68 & 106 & 22 & 203 & 37 & \\
Anthocleista djalonensis & 6,67 & 41 & 129 & 200 & 69 & \\
Phyllanthus amarus & 6,67 & 55 & 109 & 74 & 188 & \\
Nephrolepis biserrata & 6,67 & 82 & 85 & 119 & 133 & \\
Lantana camara & 6,67 & 175 & 56 & 13 & 62 & II \\
Ageratum conyzoïdes & 6,66 & 149 & 34 & 100 & 104 & \\
Mariscus alternifolius & 6,66 & 94 & 115 & 92 & 114 & \\
Clerodendrum splenden & 6,65 & 88 & 127 & 117 & 47 & \\
Heterotis rotundifolia & 6,62 & 87 & 97 & 52 & 170 & \\
Emilia praetermissa & 6,62 & 21 & 59 & 145 & 101 & \\
Phaulopsis falcisepala & 6,62 & 80 & 181 & 16 & 101 & \\
Ipomoea involucrata & 6,61 & 69 & 60 & 126 & 73 & \\
Oplismenus burmannii & 6,60 & 98 & 102 & 68 & 93 & \\
\hline Secamone afzelii & 6,54 & 86 & 152 & 9 & 138 & III \\
Panicum laxum & 6,52 & 66 & 66 & 61 & 119 & \\
\hline Sabicea venosa & 6,48 & 22 & 89 & 10 & 239 & IV \\
\hline
\end{tabular}

Les espèces d'un groupe écologique donné ont des valeurs d'information mutuelle ne différant pas les unes des autres au seuil de $5 \%$ d'après le test de Student-Fisher.

En effet, après une défriche, les espèces issues du milieu naturel d'origine sont remplacées progressivement par les taxons caractéristiques des parcelles cultivées (espèces dites rudérales). Concernant la présente étude, la présence des mêmes espèces dans les groupes écologiques caractérisant la jachère et la forêt (ou la

\section{CONCLUSION}

II ressort de l'analyse globale des profils écologiques, 3 groupes d'espèces adventices dont deux sont caractéristiques des localités étudiées. Le 1er comporte deux espèces (Lantana camara et Thaumatococcus daniellii) indicatrices des agro-écosystèmes de La Mé, en zone forestière. Le 2è est constitué de 6 espèces (Megastachya mucronata, Polygala multiflora, Anthocleista djalonensis, Asystasia gangetica, Borreria latifolia et Clerodendrum splendens) indicatrices des agro-écosystèmes de Dabou, en zone de savane incluse. savane) se justifie par le fait que la mise en jachère favorise le retour de la flore à l'état d'avant culture, assimilable à la forêt ou à la savane. En revanche, après une replantation de palmeraie, la flore sera davantage constituée d'espèces caractéristiques des milieux perturbés.

Le 3è groupe est constitué d'espèces présentant plutôt une tendance ubiquiste. Le site et le précédent cultural sont apparus les deux principaux descripteurs écologiques efficaces permettant d'expliquer la distribution des adventices. L'analyse des profils de fréquences corrigées a permis, en outre et de façon plus fine, de distinguer 3 groupes écologiques relativement aux différentes classes du descripteur "agroécosystème " et 4 autres groupes écologiques par rapport aux classes du descripteur « précédent cultural ». 


\section{RÉFÉRENCES}

Akobundu IO, 1987. Weed in the tropics. Principals and pratices. Wiley, Chichester, UK. 522 p.

Aman Kadio G, 1978. Flore et végétation des adventices dans l'hévéaculture en basse Côte d'Ivoire (Station expérimentale de I'I.R.C.A.). Étude écologique: Dynamique et structure. Thèse de Doctorat de spécialité, Malherbologie, FAST, Université d'Abidjan-Cocody, Côte d'Ivoire, 194 p.

Austin MP, 1985. Continuum concep, ordination methodes and niche theory. Annual Review of Ecology \& Systems $16: 39-61$.

Barberi P, Silvestri N and Bonari E, 1997. Weed communities of winter wheat as influenced by input level and rotation. Weed Res. 37: 301-313.

Barralis G, 1976. Méthodes d'étude des groupements adventices des cultures annuelles : application à la Côte d'Or. In: Actes du 5è Colloque International sur l'Écologie et la Biologie des Mauvaises Herbes, Dijon. Montpellier, ANPP: pp. 94-101.

Barralis $\mathrm{G}$ et Chadoeuf $\mathrm{R}, 1980$. Étude de la dynamique d'une communauté adventice : Évolution de la flore adventice au cours du cycle végétatif d'une culture. Weed Research $20:$ 231-282.

Bayer AG, 1992. Important crops of the world and their weeds (Scientific and common names, synonyms and W.S.S.A. W.S.S.J. approved computer codes). Second ed. Bayer ed., Leverkussen, Federal Republic of Germany, $1682 \mathrm{p}$.

Boraud NKM, 2000. Étude floristique et phytoécologique des adventices des complexes sucriers de Ferké 1 et 2, de Borotou-Koro et de Zuénoula, en Côte d'Ivoire. Thèse de Doctorat 3è Cycle, UFR Biosciences, Université d'Abidjan-Cocody, $157 \mathrm{p}$. + annexes.

Brown JH, 1984. On the relationship between abundance and distribution of species. Amer. Nat. 124: 255279.

Caliman JP, 1990. Dégradation des propriétés physiques conditionnant la fertilité des sols sous culture du palmier à huile en Côte d'Ivoire: Essai de correction. Thèse de Doctorat ès Sciences, Sciences du sol, Université de Bourgogne, Dijon (France), $219 \mathrm{p}$.

Caussanel JP, 1989. Nuisibilité et seuil de nuisibilité des mauvaises herbes dans une culture annuelle: relation de concurrence bispécifique. Agronomie 9: 219-240.
Daget Ph et Godron M, 1982. Analyse de l'écologie des espèces dans les communautés. Collection d'écologie. Paris, Masson, $163 \mathrm{p}$.

Déat $M$, Sément $G$ et Fontenay $P, 1980$. Premières observations sur la levée de quelques adventices tropicales. In: Actes du Vle Colloque International sur l'Écologie, la Biologie et la Systématique des mauvaises herbes (Tome 1). Montpellier, ANPP : pp. 47-56.

Déat $M$ et Touré Y, 1983. L'expérimentation d'herbicides en culture cotonnière en Côte d'Ivoire : résultats récents. In : C. R. 2è Conf. Bis. SOAM/WAWSS, Abidjan. Montpellier, SOAM/WAWSS: pp. 236247.

Debaeke $P, 1987$. Effets des systèmes de cultures sur la flore dicotylédone annuelle. Thèse de Doctorat, Sciences Agronomiques, INA-PG, Paris, France, $342 \mathrm{p}$ et annexes.

Énomyo B, 1985. Évolution des caractéristiques des sols dans les plantations de palmier à huile en zone de forêt (Côte d'Ivoire). Mémoire de fin d'études pour l'obtention du Diplôme d'Agronomie Tropicale de l'ESAT, Académie de Montpellier, France, 62 p. et annexes.

Fénelon J-P, 1999. Qu'est-ce que l'analyse des données. SEISAM, Paris, $311 \mathrm{p}$.

Fontanel P, 1987. Groupements adventices et facteurs écologiques et culturaux, comportement des espèces dans le cycle cultural, efficacité des désherbages paysans et voies d'amélioration au Sine Saloum, Sénégal. Rapport DSV/87. Montpellier, CIRAD/IRAT, $60 \mathrm{p}$.

Fontanel P, 1988. Herbicides systémiques et approche agro-écologique pour améliorer le désherbage sous les tropiques. Afr. Agric. 158: 38-40.

Forcella F and Harvey SJ, 1983. Relative abundance in an alien weed flora. Oecologia 59: 292-295.

Guillerm JL, 1971. Calcul de l'information fournie par un profil écologique et valeur indicatrice des espèces. Oecologia Plant. 6 : 209-225.

Guillerm JL, Jacquinet JC, Romane F, 1975. Tendances évolutives de la végétation spontanée du vignoble languedocien. In : Actes du Symposium Über Sukzession Forschung, Rinteln-Weser, Schmidt eds. Cramer, Vaduz : 255-267.

Hodgson JG, 1989. What is happening to the British flora? An investigation of commonness and rarity. Plants Today, 2 (1) : 26-32.

Hoffmann G, 1986. Caractérisation de la flore adventice de deux villages du terroir de Katiola (Côte 
d'Ivoire). Montpellier, IRAT-CIRAD, 54 p. et annexes.

Ipou IJ, 2005. Biologie et écologie de Euphorbia heterophylla $\mathrm{L}$ (Euphorbiaceae) en culture cotonnière, au Nord de la Côte d'Ivoire. Thèse de Doctorat, Botanique (Malherbologie) Université d'Abidjan-Cocody, $200 \mathrm{p}$.

Jackson DA, Somers KM, Harvey HH, 1989. Similarity coefficients: measures of co-occurrence and association or simply measures of occurrence? The American Nature 133 (3) : 436-453.

Karar RO, Mohamed BF, Marrs RH, 2005. Factors influencing the weed flora in the Gezira Scheme, Sudan. Weed Research 45: 121-129.

Koffi N, 1981. Étude de la levée et de la phénologie des mauvaises herbes tropicales. Cas de la zone centre de la Côte d'Ivoire. Mémoire ENSH, Protection des plantes, Versailles, France, $40 p$ et annexes.

Le Bourgeois T, 1993. Les mauvaises herbes dans la rotation cotonnière au Nord-Cameroun (Afrique centrale). Thèse de Doctorat, Biologie et Écologie Végétales, USTL, Montpellier, France, $249 p$.

Lebreton JD, Sabatier R, Banco G et Bacou AM, 1991. Principal component and correspondence analyses with respect to instrumental variables : an overview of their role in the studies of structure ; activity and species, environnement relationships : 85-114. In : Applied Multivariate Analysis in SAR and Environnemental Studies (Devillers J. \& Karcher W., eds), Brussels and Luxembourg, ECSC/ EEC/EAEC.

Legendre L, Legendre P, 1984. Écologie numérique. 1 : le traitement multiple des données écologiques (2ème éd.) Masson, Paris, $260 \mathrm{p}$.

Mahn EG and Helmecke K, 1979. Effects of herbicide treatment on the structure and functioning of agro-ecosystems. II. Structural changes in the plant community after the application of herbicides over several years. Agro-Ecosystems 5: $159-179$

Mahn EG, 1984. The influence of different nitrogen levels on the productivity and structural changes of weed communities in agro-ecosystems. In : Actes du 7è Colloque Internationale sur l'Écologie, la Biologie et Systématique des mauvaises herbes. Montpellier, ANPP : 421-429.

Maillet J, 1988. Évolution des peuplements d'adventices du vignoble du Montpelliérain. In : Actes du $8^{\text {ème }}$
Colloque Internationale sur la Biologie, l'Écologie et la Systématique des mauvaises herbes (Tome 2). Dijon, ANPP : 485-496.

Maillet J, 1992. Constitution et dynamique des communautés de mauvaises herbes des vignes de France et des rizières de Camargue. Thèse de Doctorat d'État, Biologie et Écologie végétales, USTL, Montpellier, France, $179 p$.

Malone CR, 1972. Effects of a non-selective arsenical herbicide on plant biomass and community structure in a fescue meadow. Ecology 53: 507512.

Mangara A, N'Da Adopo AA, Traoré K, Kéhé M, Soro K, Touré M, 2010. Étude phytoécologique des adventices en culture d'ananas (Ananas comosus $L$ Merr.) dans les localités de Bouna et N'Douci en Basse Côte d'Ivoire. Journal of Applied Biosciences 36 : 2367-2382.

Mark MK, 1983. Timing of seedling emergence and reproduction in some tropical dicotyledonous weeds. Weed Research $23: 325-332$.

Péné CB et Assa DA, 2003. Variations interannuelles de la pluviométrie et de l'alimentation hydrique de la canne à sucre en Côte d'Ivoire. Sécheresse 14(1) : 43-52.

Roose EJ, Fauck R, Lelong $F$ et Pédro G, 1981. Pédologie- Modifications fondamentales de la dynamique actuelle de sols ferrallitiques et ferrugineux d'Afrique Occidentale sous l'influence de la mise en culture. Comptes Rendus Académie des Sciences. Paris 292 : 1457-1460.

Sabatier R, Lebreton JD, Chessel D, 1989. Principal Componant Analysis with Instrumental Variables as a tool for modelling composition data : 341352. In: Multiway Data Analysis (Coppi R. \& Bolasco S., eds), North Holland, Amsterdam.

Tomkins DJ and Grant WF, 1977. Effects of herbicides on species diversity of two plants communities. Ecology 58 : 398-406.

Traoré $\mathrm{H}$ et Maillet J, 1998. Mauvaises herbes des cultures céréalières au Burkina Faso. Agriculture \& Développement $20: 47-59$

Traoré H, 1991. Influence des facteurs agro-écologiques sur la constitution des communautés adventices des cultures céréalières (sorgho, mil, maïs) du Burkina Faso. Thèse de Doctorat, Biologie et Écologie Végétales, USTL, Montpellier II (France), 180 p. et annexes.

Wiegleb G, 1989. Explanation and prediction in vegetation science. Vegetation 83: 17-35. 